\title{
The efficacy of pregabalin for the treatment of neuropathic pain in Japanese subjects with moderate or severe baseline pain
}

This article was published in the following Dove Medical Press journal: Journal of Pain Research

\author{
Bruce Parsons \\ Koichi Fujii ${ }^{2}$ \\ Kazutaka Nozawa ${ }^{2}$ \\ Tamotsu Yoshiyama ${ }^{3}$ \\ Marie Ortiz ${ }^{4}$ \\ Edward Whalen ${ }^{4}$ \\ 'Global Medical Product Evaluation, \\ Pfizer Inc, New York, NY, USA; \\ ${ }^{2}$ Medical Affairs, Pfizer Japan Inc, \\ Shibuya-ku, Tokyo, Japan; ${ }^{3}$ Clinical \\ Statistics, Pfizer R\&D Japan GK, \\ Shibuya-ku, Tokyo, Japan; ${ }^{4}$ Global \\ Statistics, Pfizer Inc, New York, NY, \\ USA
}

Purpose: Although analyses of pooled clinical trial data have reported how international populations respond to pregabalin by baseline neuropathic pain $(\mathrm{NeP})$ severity, no studies have evaluated this specifically in patients from Japan. Thus, this post hoc pooled analysis evaluated the efficacy of pregabalin in Japanese subjects for treating moderate or severe baseline NeP.

Patients and methods: Data were pooled from three placebo-controlled trials enrolling Japanese subjects with postherpetic neuralgia (PHN), diabetic peripheral neuropathy (DPN), and spinal cord injury (SCI). The efficacy of pregabalin was evaluated by baseline pain severity (moderate or severe NeP). The trials on PHN and DPN included a 1-week titration of pregabalin from $150 \mathrm{mg} /$ day to 300 or $600 \mathrm{mg}$ /day; the SCI trial included a 4-week dose optimization phase (150 mg/day, titrated up to $600 \mathrm{mg}$ /day). Treatment durations were 13-16 weeks (excluding 1-week taper periods), and pregabalin was administered in two divided doses per day.

Results: Mean baseline pain scores and demographic characteristics were comparable between treatment cohorts. Pregabalin treatment significantly reduced pain scores from baseline to endpoint compared with placebo in subjects with both moderate $(P<0.001)$ and severe $(P<0.05)$ baseline pain. Significant improvements in mean sleep scores from baseline to endpoint were associated with pregabalin compared with placebo in subjects with both moderate and severe baseline pain (both $P<0.0001$ ). A greater proportion of subjects in both pain cohorts achieved a $\geq 30 \%$ reduction in pain from baseline with pregabalin vs placebo $(P<0.05)$. Higher proportions of pregabalin-treated vs placebo-treated subjects shifted to a less severe pain category at endpoint. Consistent with the known safety profile of pregabalin, common adverse events included dizziness, somnolence, weight gain, and peripheral edema.

Conclusion: Pregabalin demonstrated efficacy for pain relief and sleep improvement with a consistent safety profile in Japanese subjects with either moderate or severe baseline pain severity Clinical Trials gov identifiers: NCT0039490130, NCT0055347522, NCT0040774524

Keywords: diabetic peripheral neuropathy, postherpetic neuralgia, spinal cord injury, chronic pain, sleep

\section{Introduction}

Neuropathic pain $(\mathrm{NeP})$ is defined as pain caused by a lesion or disease of the somatosensory nervous system. NeP is associated with conditions such as postherpetic neuralgia (PHN), diabetic peripheral neuropathy (DPN), and spinal cord injury (SCI) ${ }^{1,2}$ Chronic NeP can significantly reduce quality-of-life (QoL) and can impose a considerable economic burden on individual patients, their families, and society. ${ }^{3}$

According to two surveys conducted in nationwide samples of the general Japanese population, the prevalence of NeP in Japan is estimated to be $3.2 \%{ }^{4,5}$ Although most research on the health and economic burdens of NeP conditions focuses on Western
Global Medical Product Evaluation, Pfizer Inc, 235 East 42nd Street,

New York, NY 10017, USA

Tel +I 2125731649

Email bruce.parsons@pfizer.com 
populations, available research in Japan indicates a considerable burden on the general population. Japanese patients with NeP have been shown to experience poorer QoL, higher psychological distress, greater sleep disturbance, and loss of work productivity compared with the general population. ${ }^{4,6}$

A consistent finding among both Western and Asian populations is that most patients with NeP report the intensity of chronic pain as "moderate" or "severe" rather than as "mild". ${ }^{7-11}$ Indeed, the burden of NeP is heavier in patients with more severe, chronic pain. ${ }^{10,12-14}$ In addition, among patients diagnosed with a range of $\mathrm{NeP}$ conditions in routine clinical practice in the US, a significant association was found between pain severity and economic burden, with the highest costs incurred by patients with severe pain. Across $\mathrm{NeP}$ categories, high pain severity was associated with a heavier burden of comorbidities, increased use of healthcare resources and NeP medications, greater NeP-associated productivity loss, and considerable direct and indirect costs. In a multinational survey of subjects with DPN, reports from Asia, Latin America, and the Middle East concurred regarding difficulties in functioning, sleep, and general health status. Difficulties increased in tandem with pain severity, supporting the view that DPN, like other NeP conditions, is often inadequately managed and imposes a burden worldwide. ${ }^{14}$

Pregabalin, an $\alpha 2-\delta$ ligand with analgesic properties, is an approved treatment option for $\mathrm{NeP}$ associated with $\mathrm{PHN}$, DPN, and SCI in the US, and for the treatment of peripheral and central NeP in Europe and Japan. ${ }^{15,16}$ Pregabalin is also indicated for the management of fibromyalgia in the US and for the management of pain associated with fibromyalgia in Japan. ${ }^{17}$ The analgesic efficacy and safety of pregabalin in patients with $\mathrm{NeP}$ have been demonstrated in clinical trials conducted worldwide, including in the US, Europe, China, Japan, and Korea. ${ }^{18-30}$ Pregabalin studies have also highlighted the importance of tailoring treatments to individual needs in order to improve the overall effectiveness of treatment regimens. Data pooled from 16 pregabalin trials in subjects with PHN or DPN suggest that the presence of comorbid sleep disturbance could predict pain relief in response to pregabalin. ${ }^{31}$ An analysis of pooled data from 11 pregabalin trials in DPN demonstrated that subjects with severe baseline pain experienced greater improvements in both pain relief and sleep disturbance than subjects with moderate pain. ${ }^{32}$

Pooled analyses of global clinical trial data have shown that, in general, patients with moderate or severe baseline NeP respond well to pregabalin. ${ }^{31,32}$ However, because different regions and races can have distinct cultural and biological factors that may impact treatment responses, one should not assume that clinical evidence in one population would apply to another. To determine whether the previous international study findings are also evident in Japanese patients with NeP, the current study sought to demonstrate the therapeutic effects of pregabalin in subjects from Japan reporting moderate or severe baseline pain.

\section{Materials and methods Study design}

The three published randomized, double-blind, placebocontrolled, multicenter pregabalin trials are registered on ClinicalTrials.gov (sponsor study \#/ClinicalTrials.gov identifier: PHN study A0081120/NCT00394901, ${ }^{30,33}$ DPN study A0081163/NCT00553475, 22,30 and SCI study A0081107/ NCT00407745). ${ }^{24}$ The PHN (September 2006-November 2007) and DPN (October 2007-March 2009) trials were conducted in Japan, and the SCI trial (January 2007-February 2011) was conducted worldwide, but only data from the Japanese subpopulation were selected for the current analysis.

The PHN and DPN trials included a 1-week titration period, during which subjects received pregabalin $150 \mathrm{mg} /$ day, titrated to $300 \mathrm{mg} /$ day or $600 \mathrm{mg} /$ day. The SCI trial included a 4-week dose optimization period, during which subjects received pregabalin $150 \mathrm{mg}$ /day for 1 week, with titration up to $600 \mathrm{mg} /$ day based on efficacy and tolerability. All trials included a placebo arm. The doses administered in all three trials were divided into two equal doses per day. Although treatment duration ranged from 13-16 weeks, excluding the taper periods (Table 1), all trials included a fixed-dose/maintenance period of 12 weeks.

For all trials, subjects were selected for being aged $\geq 18$ years and having a mean daily pain score $\geq 4$ over a 7 -day baseline period on an 11-point numeric rating scale (NRS, where $0=$ no pain and $10=$ worst possible pain). In the PHN and DPN studies, eligible subjects were required to score $\geq 40$ $\mathrm{mm}$ on the $0-100 \mathrm{~mm}$ visual analog scale of the Short-Form McGill Pain Questionnaire at screening and randomization. The following primary diagnoses applied: 1) PHN (pain persisting $\geq 3$ months after the healing of the herpes zoster rash); 2) DPN (history of type I or II diabetes mellitus for $\geq 1$ year, with painful distal, symmetrical, sensorimotor polyneuropathy); and 3) SCI (complete or incomplete lesion $\geq 12$-month duration at $\mathrm{C} 2-\mathrm{T} 12$ vertebrae).

The primary efficacy measure for the PHN and DPN studies was the endpoint mean pain score for the 7 days preceding study completion. In the SCI study, the primary endpoint was duration-adjusted average change based on 
Table I Summary of neuropathic pain studies included in the analysis

\begin{tabular}{|c|c|c|c|c|c|c|}
\hline \multirow{2}{*}{$\begin{array}{l}\text { Condition (study numberl } \\
\text { ClinicalTrials.gov identifier) }\end{array}$} & \multirow{2}{*}{$\begin{array}{l}\text { Total treatment } \\
\text { phase/maintenance } \\
\text { phase (weeks) }\end{array}$} & \multirow{2}{*}{$\begin{array}{l}\text { Pregabalin } \\
\text { maintenance } \\
\text { dose (mg/day) }\end{array}$} & \multirow[t]{2}{*}{ Administration } & \multicolumn{3}{|c|}{ No. of participants ${ }^{a}$} \\
\hline & & & & Placebo & Pregabalin & Total \\
\hline PHN (A008I I 20/NCT0039490I) $)^{30,34}$ & $13 / 12$ & $150,300,600^{b}$ & BID fixed dose & 97 & 272 & 369 \\
\hline DPN (A008II63/NCT00553475) & $13 / 12$ & $300,600^{\mathrm{b}}$ & BID fixed dose & 135 & 179 & 314 \\
\hline SCl (A008II07/NCT00407745) $)^{24}$ & $16 / 12$ & $150-600$ & BID flexible dose & 27 & 32 & 59 \\
\hline
\end{tabular}

Notes: aEfficacy analyses were performed on all randomized subjects with baseline mean pain score $\geq 4$ who had received at least one dose of study medication, with at least one post-baseline entry in the daily pain diary for each trial. Efficacy analysis for the $\mathrm{SCl}$ trial was based on the Japanese subpopulation. ${ }^{\mathrm{b}} \mathrm{All}$ trials included a matching placebo treatment arm.

Abbreviations: BID, twice-daily; DPN, diabetic peripheral neuropathy; PHN, post-herpetic neuralgia; SCl, spinal cord injury.

pain scores recorded during the entire treatment period. Secondary efficacy endpoints included endpoint mean sleep interference score (sleep score) derived from the daily sleep diary (11-point NRS). The patient global impression of change questionnaire (PGIC) was also assessed at the end of the trial (where $1=$ very much improved and $7=$ very much worse). The incidence, severity, and relationship to study treatment of adverse events (AEs) were recorded for safety and tolerability assessments.

All trials included in the current analysis were approved by the appropriate institutional review boards or independent ethics committees at each investigational center. All trials were conducted in compliance with the ethical principles originating in or derived from the Declaration of Helsinki and in compliance with International Conference on Harmonization Good Clinical Practice guidelines. All participants provided written consent before entering a trial.

\section{Statistical analysis}

Subjects who received at least one dose of study medication and who had a baseline pain score $>4$ were included in the pooled efficacy and safety analysis. For all three trials, data from the pregabalin and placebo treatment groups were extracted into a single pregabalin-treatment and a single placebo-treatment group, respectively. Subjects were subgrouped by baseline pain scores into severity categories of moderate (NRS pain score $\geq 4-<$ ) or severe $(\geq 7-10)$ pain. Demographics and baseline characteristics and the incidence of AEs were summarized by means of descriptive statistics.

Mean changes in pain and sleep interference scores at endpoint were analyzed using an analysis of variance model. For pain, the terms used were baseline pain severity, treatment, and protocol, and the interaction terms were treatment by baseline pain severity and treatment by protocol by baseline pain severity; protocol was used instead of indication. For sleep, similar interaction terms as in the pain severity analyses were used, but the terms also included baseline sleep score. Pain responders were defined as subjects with $\geq 30 \%$ or $\geq 50 \%$ reductions in mean pain score from the start of treatment to endpoint. PGIC responders were defined as subjects who were "much improved" or "very much improved". Pain and PGIC responder status were analyzed by a logistic regression model using terms for treatment and baseline pain severity, and terms for interaction between treatment and baseline severity. The percentage of subjects shifting from severe or moderate pain at baseline to mild, moderate, or severe pain at endpoint was calculated for each treatment arm. The last observation carried forward approach, commonly used in handling missing data for continuous measures, was used in efficacy analyses. The modified baseline carried forward approach, widely accepted to account for discontinuations due to treatment-related causes, was used for pain response endpoint analyses.

\section{Results}

\section{Demographics}

The study cohort consisted of 742 Japanese subjects with NeP. Most subjects reported moderate $(\mathrm{n}=513$ [69.1\%]) rather than severe baseline pain (Table 2). The majority of subjects were male (65.2\%), and median age ranged from 65-69 years. Within the moderate and severe pain cohorts, baseline mean pain scores were comparable between the pregabalin and placebo treatment groups (Table 2). Higher sleep scores were reported in the severe pain cohort compared with the moderate pain cohort.

\section{Efficacy}

Pregabalin treatment significantly reduced pain scores from baseline to endpoint compared with placebo in subjects with moderate $(P<0.001)$ or severe $(P<0.05)$ pain (Table 3$)$. A pronounced shift in pain burden was evident at therapeutic dose levels for PHN and DPN among patients with moderate or severe baseline pain (Figure 1A and B). 
Table 2 Baseline demographic and clinical characteristics

\begin{tabular}{|c|c|c|c|c|}
\hline & \multicolumn{4}{|c|}{ NeP population ${ }^{\mathrm{a}}$ : baseline pain ${ }^{\mathrm{b}}$} \\
\hline & \multicolumn{2}{|c|}{ Moderate } & \multicolumn{2}{|l|}{ Severe } \\
\hline & Pregabalin & Placebo & Pregabalin & Placebo \\
\hline $\mathrm{N}$ & 333 & 180 & 150 & 79 \\
\hline Age, years, median (range) & $66(24-92)$ & $65(28-88)$ & $69(27-88)$ & $68(27-87)$ \\
\hline \multicolumn{5}{|l|}{ Sex, n (\%) } \\
\hline Female & $118(35.4)$ & $52(28.9)$ & $66(44.0)$ & $22(27.9)$ \\
\hline Male & $215(64.6)$ & $128(7 \mid . I)$ & $84(56.0)$ & $57(72.2)$ \\
\hline Duration of NeP-related pain, months, mean (SD) & $44.2(50.4)$ & $47.2(45.9)$ & $53.3(65.9)$ & $49.7(49.3)$ \\
\hline Pain score, ${ }^{\mathrm{b}}$ mean (SD) & $5.4(0.8)$ & $5.4(0.8)$ & $8.0(0.9)$ & $7.9(0.8)$ \\
\hline Sleep score, ${ }^{\mathrm{c}}$ mean (SD) & $3.2(2.1)$ & $3.5(2.0)$ & $5.3(2.8)$ & $5.4(2.8)$ \\
\hline
\end{tabular}

Notes: Subjects with baseline pain $<4$ and missing baseline pain scores are excluded. alncludes pregabalin 150, 300, 600 mg/day, and flexible $150-600$ mg/day treatment

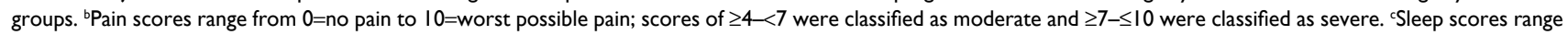
from $0=$ pain does not interfere with sleep to $10=$ pain completely interferes with sleep.

Abbreviation: NeP, neuropathic pain.

Table 3 Change in mean pain and sleep scores from baseline to endpoint (LOCF analysis)

\begin{tabular}{|l|l|l|l|l|l|l|}
\hline & \multicolumn{2}{|l|}{ Pregabalin } & \multicolumn{2}{l|}{ Placebo } & Difference from placebo \\
\cline { 2 - 7 } & $\mathbf{n}$ & LS mean change (SE) & $\mathbf{n}$ & LS mean change (SE) & LS mean difference (SE) & P-value \\
\hline Change in pain score \\
\hline
\end{tabular}

Notes: ANOVA model includes baseline pain severity, treatment, and indication (study). Sleep model also included baseline sleep score. Second interaction term included treatment, indication, and baseline pain severity. Subjects with baseline pain $<4$ and missing baseline pain scores are excluded. ${ }^{2}$ Pain scores range from $0=$ no pain to $10=$ worst possible pain; scores of $\geq 4-<7$ were classified as moderate and $\geq 7-\leq 10$ were classified as severe. IIncludes pregabalin I50, 300, 600 mg/day, and flexible $150-600 \mathrm{mg} / \mathrm{day}$ treatment groups. Sleep scores range from $0=$ pain does not interfere with sleep to $10=$ pain completely interferes with sleep.

Abbreviations: LOCF, last observation carried forward; LS, least squares; NeP, neuropathic pain.

In the analysis of change from baseline to endpoint in mean sleep scores, significant improvement $(P<0.0001)$ was associated with pregabalin vs placebo treatment in subjects with moderate and severe baseline pain (Table 3). A greater proportion of subjects with $\mathrm{NeP}$ achieved a $\geq 30 \%$ responder status for pain with pregabalin vs placebo in both pain severity cohorts $(P<0.05)$ (Table 4$)$. Although the proportion of subjects with $\mathrm{NeP}$ who achieved $\geq 50 \%$ pain responder status in both baseline pain severity cohorts was numerically greater in subjects treated with pregabalin than with placebo, the difference reached significance only in the severe pain cohort $(P<0.05)$. Pregabalin significantly improved the proportion of PGIC responders (score $\leq 2$ ) at endpoint in both the moderate pain $(P<0.05)$ and severe pain $(P<0.01)$ cohorts (Figure 2$)$.

Treatment with pregabalin successfully shifted $20 \%-30 \%$ of Japanese subjects with NeP across both levels of baseline pain severity to a category of lesser severity (Figure 1). In addition, greater proportions of subjects with severe pain at baseline shifted two pain levels (from severe to mild pain) at endpoint after treatment with pregabalin vs placebo.

\section{Adverse events}

Overall, $85 \%-87 \%$ of pregabalin-treated subjects in the moderate and severe baseline pain cohorts experienced at least one treatment-emergent AE (TEAE) compared with $66 \%-71 \%$ of placebo-treated subjects (Table 5). Common AEs (reported in $\geq 10 \%$ of pregabalin-treated subjects and at twice the rate seen with placebo) observed in $\mathrm{NeP}$ subjects were consistent with the known safety profile of pregabalin and included dizziness, somnolence, weight gain, and peripheral edema.

Among pregabalin-treated subjects, 96 permanently withdrew from the studies because of TEAEs, of which 69 events were judged to be treatment-related by the investigators. Treatment-related AEs were cited in 4.7\%, 12.4\%, 
A

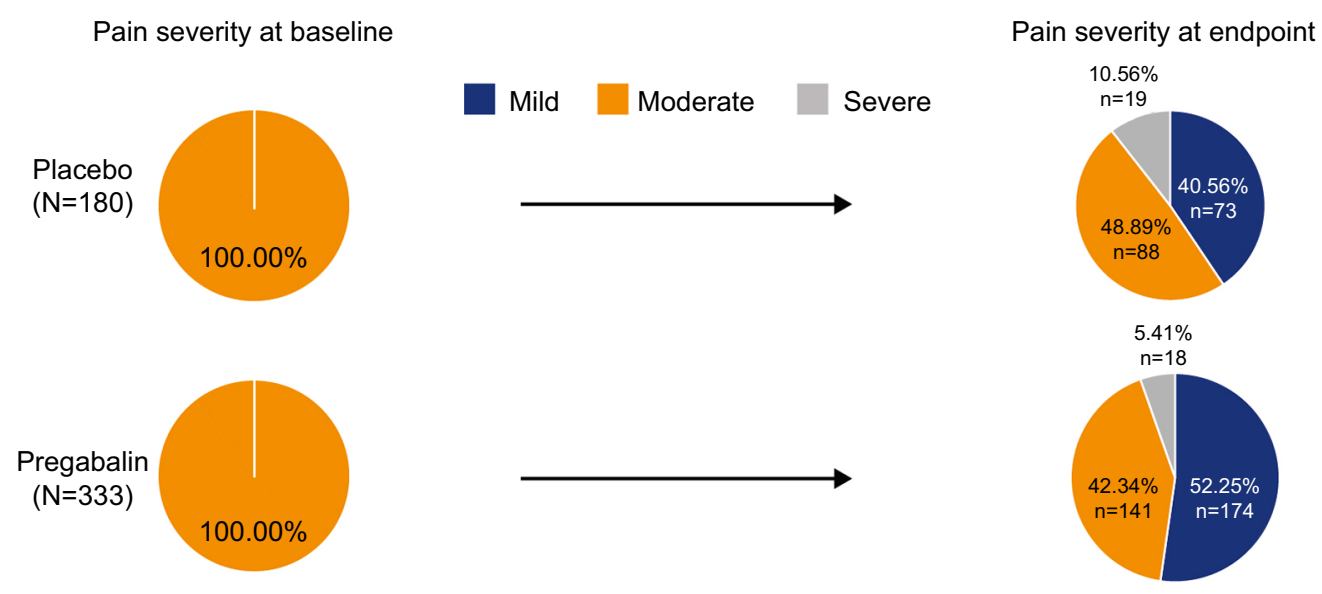

B

Pain severity at baseline

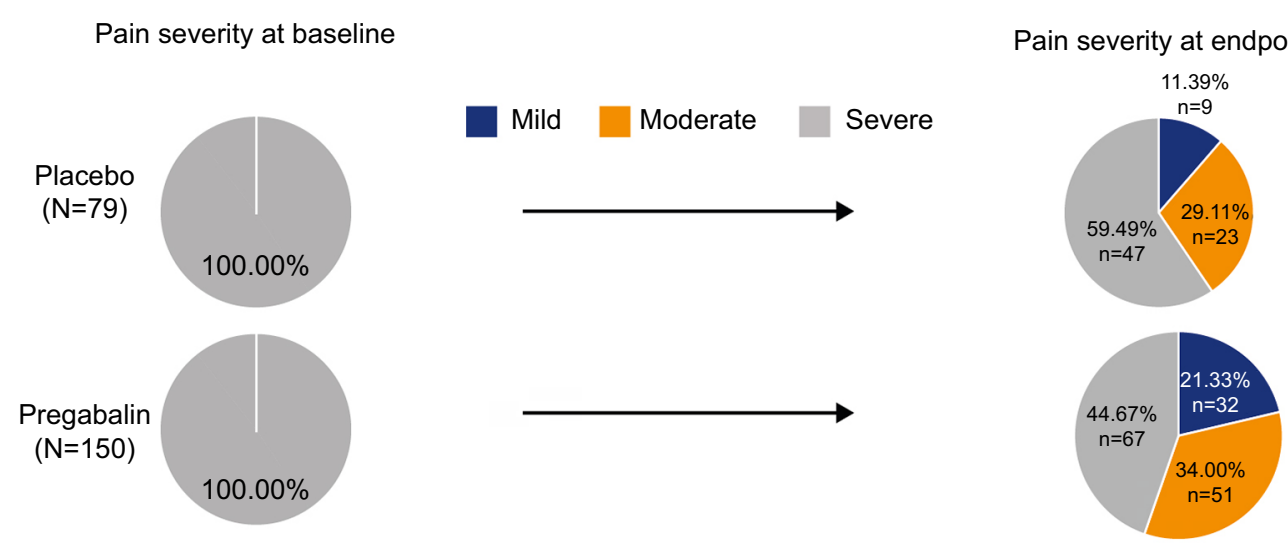

Pain severity at endpoint

Figure I Pain shift from baseline to endpoint for mean pain score by $(\mathbf{A})$ moderate $(\geq 4-<7)$ and $(\mathbf{B})$ severe $(\geq 7-\leq 10)$ baseline pain criteria in pregabalin and placebo treatment groups.

Notes: Population: subjects in pregabalin studies NCT0039490I (PHN), NCT00553475 (DPN), and modified intent-to-treat Japanese subjects in pregabalin study NCT00407745 (SCl). Percentages are based on $n$ for each treatment; $n$, total number of subjects with baseline mean pain $\geq 4-\leq 10$.

Abbreviations: DPN, diabetic peripheral neuropathy; PHN, post-herpetic neuralgia; SCl, spinal cord injury.

Table 4 Analysis of $30 \%$ and $50 \%$ pain responders at $\mathrm{mBOCF}$ endpoint in $\mathrm{NeP}$

\begin{tabular}{|c|c|c|c|c|c|}
\hline \multirow[b]{2}{*}{ All pregabalin } & \multirow[b]{2}{*}{ Pregabalin responders, $\mathbf{n}(\%)$} & \multirow[b]{2}{*}{ Placebo responders, n (\%) } & \multicolumn{3}{|c|}{ vs placebo } \\
\hline & & & OR & $95 \% \mathrm{Cl}$ & $P$-value \\
\hline \multicolumn{6}{|c|}{$30 \%$ pain responders ${ }^{a}$} \\
\hline Moderate $^{\mathrm{b}}$ & $154 / 333(46.3)$ & $63 / 180(35.0)$ & 1.60 & $1.10-2.32$ & 0.014 \\
\hline Severe $^{b}$ & $48 / 150(32.0)$ & $15 / 79(19.0)$ & 2.01 & $1.04-3.88$ & 0.038 \\
\hline \multicolumn{6}{|c|}{$50 \%$ pain responders ${ }^{a}$} \\
\hline Moderate $^{b}$ & $95 / 333(28.5)$ & $38 / 180(21.1)$ & 1.49 & $0.97-2.29$ & 0.068 \\
\hline Severe $^{\mathrm{b}}$ & $32 / 150(21.3)$ & $7 / 79(8.9)$ & 2.79 & $1.17-6.65$ & 0.021 \\
\hline
\end{tabular}

Notes: Subjects with baseline pain $<4$ and missing baseline pain scores are excluded. NeP population includes pregabalin I50, 300, $600 \mathrm{mg} / \mathrm{day}$, and flexible I50-600 mg/day treatment groups. Logistic regression model used with terms for treatment and baseline pain severity (either moderate or severe) and interaction terms of treatment and baseline severity as the categorical covariates. ${ }^{\text {PPain }}$ responders were subjects with $\geq 30 \%$ or $\geq 50 \%$ reduction in mean pain score from baseline to endpoint. ${ }^{b}$ Baseline pain scores were defined as moderate $(\geq 4-7)$ or severe $(\geq 7-10)$.

Abbreviation: mBOCF, modified baseline carried forward approach; NeP, neuropathic pain; OR, odds ratio.

and $18.9 \%$ of study discontinuations from the groups given pregabalin 150, 300, and $600 \mathrm{mg} /$ day, respectively, and in $9.4 \%$ of those who received pregabalin flexible dosing. The highest rate of discontinuation from study was observed at the maximum pregabalin dose level. Treatment-related TEAEs were cited in $3.9 \%$ of permanent discontinuations by placebo-treated subjects. Only one subject, in the pregabalin $150 \mathrm{mg} /$ day group, permanently discontinued study because 
of a laboratory abnormality. Lack of efficacy was the reason given for $4.7 \%, 1.5 \%$, and $0.9 \%$ of permanent discontinuations from the pregabalin 150,300, and $600 \mathrm{mg} /$ day groups, but not in the flexible-dose group. Overall, 15.1\%, 20.1\%, $24.5 \%$, and $15.6 \%$ of subjects in the pregabalin 150,300, 600 $\mathrm{mg} /$ day, and flexible-dose groups discontinued pregabalin, whereas $13.5 \%$ of subjects discontinued placebo.

\section{Discussion}

In this current pooled analysis in Japanese subjects with $\mathrm{NeP}$, pregabalin was efficacious in improving pain relief, sleep quality, and PGIC scores, regardless of baseline pain severity. Pregabalin was well tolerated in this patient population.

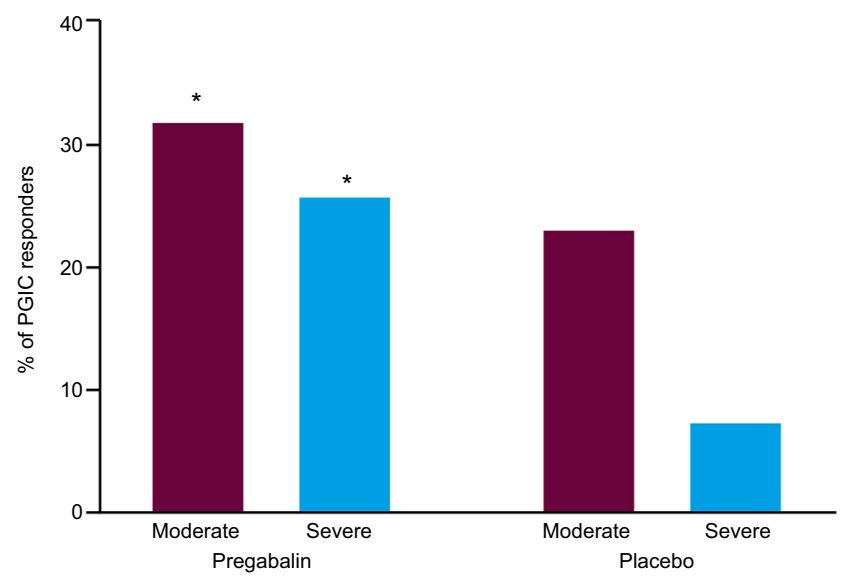

Figure 2 PGIC responders at endpoint by baseline pain severity. Notes: $* P<0.05$. PGIC responders were subjects who scored "much improved" $($ score $=2)$ or "very much improved" (score $=1$ ) on a scale of I-7. Baseline pain scores were defined as moderate $(\geq 4-<7)$ or severe $(\geq 7-10)$. Logistic regression model used with terms for treatment and baseline pain severity (moderate or severe) and interaction term of baseline severity and treatment as the categorical covariates. Abbreviation: PGIC, patient global impression of change.
The therapeutic efficacy of pregabalin for reducing mean pain and sleep scores at endpoint appeared to be comparable between subjects with severe and moderate baseline pain (Table 3). Although there were numerical differences in the odds of achieving $30 \%$ pain responder status at endpoint between subjects with baseline severe pain compared with moderate pain, the overlapping 95\% CIs suggested that the odds were not meaningfully different, regardless of pain severity. This is in contrast to conclusions from previous international pooled studies, in which subjects with severe baseline pain experienced greater therapeutic benefits with pregabalin than did subjects with moderate baseline pain. ${ }^{32}$ Consistent with that study, however, subjects in the current study with severe baseline $\mathrm{NeP}$ had significantly higher odds (vs placebo) to be $50 \%$ pain responders at endpoint, but subjects with moderate baseline pain only demonstrated numerically (but not statistically significantly) higher odds than placebo. Additional research would be warranted to explore the aforementioned differences between the previous global study and this study conducted in Japan (eg, what impact do cultural and biological differences have on these outcomes?).

Subjects with severe pain at baseline "shifted" to mild pain at nearly twice the frequency after treatment with pregabalin (21.33\%) compared with placebo (11.39\%). Overall, $20 \%-30 \%$ of Japanese subjects with NeP who had moderateto-severe pain at baseline had improved to experiencing mildto-moderate pain after 13-16 weeks of pregabalin treatment.

Overall improvement in global health status appeared to be comparable regardless of baseline pain severity, as observed by the proportion of PGIC responders at endpoint being comparable between subjects with moderate and severe baseline pain. The incidence of commonly experienced AEs

Table 5 Summary of treatment-emergent AEs (all-causality) by baseline pain severity in neuropathic pain

\begin{tabular}{|c|c|c|c|c|}
\hline \multirow[b]{3}{*}{ Subjects with AEs', n (\%) } & \multicolumn{4}{|c|}{ Baseline pain $^{a}$} \\
\hline & \multicolumn{2}{|l|}{ Moderate } & \multicolumn{2}{|l|}{ Severe } \\
\hline & $\begin{array}{l}\text { Pregabalin } \\
(n=333)\end{array}$ & $\begin{array}{l}\text { Placebo } \\
(n=180)\end{array}$ & $\begin{array}{l}\text { Pregabalin } \\
(n=150)\end{array}$ & Placebo $(n=79)$ \\
\hline Subjects with $\geq I A E$ & $282(84.7)$ & $127(70.6)$ & $13 \mid(87.3)$ & $52(65.8)$ \\
\hline \multicolumn{5}{|l|}{ Common AEs ${ }^{c}$} \\
\hline Dizziness & $99(29.7)$ & $11(6.1)$ & $39(26.0)$ & $6(7.6)$ \\
\hline Somnolence & $98(29.4)$ & $20(\mathrm{II} . \mathrm{I})$ & $43(28.7)$ & $2(2.5)$ \\
\hline Weight gain & $44(13.2)$ & $3(1.7)$ & $15(10.0)$ & $3(3.8)$ \\
\hline Constipation & $32(9.6)$ & $6(3.3)$ & $13(8.7)$ & $3(3.8)$ \\
\hline Peripheral edema & $44(13.2)$ & $6(3.3)$ & $15(10.0)$ & $2(2.5)$ \\
\hline
\end{tabular}

Notes: Subjects with baseline pain $<4$ and missing baseline pain scores are excluded. ${ }^{\text {aB }}$ Baseline pain scores defined as moderate $(\geq 4-7)$ or severe $(\geq 7-10)$. ${ }^{b}$ Defined by Medical Dictionary of Regulatory Activities preferred terms. 'Reported in $\geq 5 \%$ of subjects and at twice the rate of placebo within a disease cohort.

Abbreviation: $A E$, adverse event. 
(dizziness, somnolence, weight gain, and peripheral edema) with pregabalin was generally low, and consistent with its known safety profile. ${ }^{15-17}$

\section{Limitations}

A few limitations of the current analysis need to be considered. First, fewer subjects were drawn from the SCI study than from the PHN and DPN studies. In addition, pain and sleep efficacy assessments relied on patient diary entries, which, despite being a standard procedure in the evaluation of analgesic efficacy, could possibly introduce self-reporting bias. Finally, this is a post hoc pooled analysis of clinical trial data and, as such, these analyses were not prespecified in the original study designs of the source trials.

\section{Conclusion}

In this analysis, pregabalin (compared with placebo) was efficacious in reducing mean pain score and improving sleep in Japanese subjects with either moderate or severe NeP at baseline. In addition, both pain severity groups had significant odds of achieving 30\% pain responder status, and the severe baseline pain group of becoming $50 \%$ pain responders. Both baseline pain severity groups shifted to lower categories of pain (eg, severe to moderate, severe to mild, moderate to mild) at a frequency higher than placebo. Although numerical differences in the above parameters were observed between subjects with severe vs moderate baseline pain, the overlapping error values suggested these differences were not meaningful. Finally, pregabalin was generally well tolerated in these subjects. These findings appear to support the premise that pregabalin is an efficacious treatment for improving pain and sleep, irrespective of pain severity in subjects in Japan.

\section{Acknowledgments}

The original studies and the current manuscript were funded by Pfizer. Editorial/medical writing support was provided by Penny Gorringe, MSc, of Engage Scientific Solutions, and was funded by Pfizer. Upon request, and subject to certain criteria, conditions, and exceptions (see https://www.pfizer.com/science/clinical-trials/trial-data-and-results for more information), Pfizer will provide access to individual de-identified participant data from Pfizer-sponsored global interventional clinical studies conducted for medicines, vaccines, and medical devices; 1) for indications that have been approved in the US and/or EU or 2) in programs that have been terminated (ie, development for all indications has been discontinued). Pfizer will also consider requests for the protocol, data dictionary, and statistical analy- sis plan. Data may be requested from Pfizer trials 24 months after study completion. The de-identified participant data will be made available to researchers whose proposals meet the research criteria and other conditions, and for which an exception does not apply, via a secure portal. To gain access, data requestors must enter into a data access agreement with Pfizer.

\section{Author contributions}

All authors contributed to the interpretation of the data, drafting and revising the article, gave final approval of the version to be published, and agree to be accountable for all aspects of the work. BP, KF, KN, and TY were involved in the study design. TY was also involved in the acquisition of the data. $\mathrm{KF}, \mathrm{KN}, \mathrm{EW}$, and MO conducted the analyses of the data.

\section{Disclosure}

BP, TY, KN, KF, MO, and EW are full-time employees of, and own stock in Pfizer. The authors report no other conflicts of interest in this work.

\section{References}

1. International Association for the Study of Pain. IASP taxonomy: pain terms; 1994. Available from: https://www.iasp-pain.org/Taxonomy\#Pe ripheralneuropathicpain. Accessed March 30, 2018.

2. Gilron I, Baron R, Jensen T. Neuropathic pain: principles of diagnosis and treatment. Mayo Clin Proc. 2015;90(4):532-545.

3. Doth AH, Hansson PT, Jensen MP, Taylor RS. The burden of neuropathic pain: a systematic review and meta-analysis of health utilities. Pain 2010;149(2):338-344.

4. Inoue S, Taguchi T, Yamashita T, Nakamura M, Ushida T. The prevalence and impact of chronic neuropathic pain on daily and social life: a nationwide study in a Japanese population. Eur J Pain. 2017;21(4):727-737.

5. Nakamura M, Nishiwaki Y, Sumitani M, et al. Investigation of chronic musculoskeletal pain (third report): with special reference to the importance of neuropathic pain and psychogenic pain. J Orthop Sci. 2014;19(4):667-675.

6. Yamashita T, Takahashi K, Yonenobu K, Kikuchi S. Prevalence of neuropathic pain in cases with chronic pain related to spinal disorders. J Orthop Sci. 2014;19(1):15-21.

7. van Seventer R, Sadosky A, Lucero M, Dukes E. A cross-sectional survey of health state impairment and treatment patterns in patients with postherpetic neuralgia. Age Ageing. 2006;35(2):132-137.

8. Davies M, Brophy S, Williams R, Taylor A. The prevalence, severity, and impact of painful diabetic peripheral neuropathy in type 2 diabetes. Diabetes Care. 2006;29(7):1518-1522.

9. Sadosky A, Schaefer C, Mann R, et al. Burden of illness associated with painful diabetic peripheral neuropathy among adults seeking treatment in the US: results from a retrospective chart review and cross-sectional survey. Diabetes Metab Syndr Obes. 2013;6:79-92.

10. Schaefer C, Chandran A, Hufstader M, et al. The comparative burden of mild, moderate and severe fibromyalgia: results from a cross-sectional survey in the United States. Health Qual Life Outcomes. 2011;9(1):71.

11. Mann R, Schaefer C, Sadosky A, et al. Burden of spinal cord injuryrelated neuropathic pain in the United States: retrospective chart review and cross-sectional survey. Spinal Cord. 2013;51(7):564-570.

12. Schaefer C, Sadosky A, Mann R, et al. Pain severity and the economic burden of neuropathic pain in the United States: BEAT neuropathic pain observational study. Clinicoecon Outcomes Res. 2014;6:483-496. 
13. DiBonaventura MD, Cappelleri JC, Joshi AV. Association between pain severity and health care resource use, health status, productivity and related costs in painful diabetic peripheral neuropathy patients. Pain Med. 2011;12(5):799-807.

14. Hoffman DL, Sadosky A, Alvir J. Cross-national burden of painful diabetic peripheral neuropathy in Asia, Latin America, and the Middle East. Pain Pract. 2009;9(1):35-42.

15. European Medicines Agency. EPAR summary for the public: pregabalin Pfizer. EMA/220635/2014; 2014. Available from: http://www.ema. europa.eu/docs/en_GB/document_library/EPAR_-_Summary_for_the_ public/human/003880/WC500166174.pdf. Accessed March 30, 2018.

16. Pfizer Japan Inc, Eisai Co Ltd. Pfizer Japan release: Launch of pain treatment Lyrica OD tablet formulation in Japan; 2017. Available from: https:/www. biospace.com/article/releases/pfizer-japan-release-launch-of-pain-treatmentlyrica-od-tablet-formulation-in-japan-/. Accessed March 30, 2018.

17. Pfizer Inc. Lyrica (pregabalin) prescribing information; 2004. Available from: http://labeling.pfizer.com/ShowLabeling.aspx?id=561. Accessed March 30, 2018.

18. Rosenstock J, Tuchman M, LaMoreaux L, Sharma U. Pregabalin for the treatment of painful diabetic peripheral neuropathy: a double-blind, placebo-controlled trial. Pain. 2004;110(3):628-638.

19. Lesser H, Sharma U, LaMoreaux L, Poole RM. Pregabalin relieves symptoms of painful diabetic neuropathy: a randomized controlled trial. Neurology. 2004;63(11):2104-2110.

20. Richter RW, Portenoy R, Sharma U, LaMoreaux L, Bockbrader H, Knapp LE. Relief of painful diabetic peripheral neuropathy with pregabalin: a randomized, placebo-controlled trial. J Pain. 2005;6(4):253-260.

21. Arezzo JC, Rosenstock J, LaMoreaux L, Pauer L. Efficacy and safety of pregabalin $600 \mathrm{mg} / \mathrm{d}$ for treating painful diabetic peripheral neuropathy: a double-blind placebo-controlled trial. BMC Neurol. 2008;8:33.

22. Satoh J, Yagihashi S, Baba M, et al. Efficacy and safety of pregabalin for treating neuropathic pain associated with diabetic peripheral neuropathy: a 14 week, randomized, double-blind, placebo-controlled trial. Diabet Med. 2011;28(1):109-116.

23. Tölle T, Freynhagen R, Versavel M, Trostmann U, Young JP. Pregabalin for relief of neuropathic pain associated with diabetic neuropathy: a randomized, double-blind study. Eur J Pain. 2008;12(2):203-213.

24. Cardenas DD, Nieshoff EC, Suda K, et al. A randomized trial of pregabalin in patients with neuropathic pain due to spinal cord injury. Neurology. 2013;80(6):533-539.
25. Siddall PJ, Cousins MJ, Otte A, Griesing T, Chambers R, Murphy TK. Pregabalin in central neuropathic pain associated with spinal cord injury: a placebo-controlled trial. Neurology. 2006;67(10):1792-1800.

26. Mu Y, Liu X, Li Q, et al. Efficacy and safety of pregabalin for painful diabetic peripheral neuropathy in a population of Chinese patients: a randomized placebo-controlled trial. J Diabetes. 2018;10(3): 256-265.

27. Moon DE, Lee DI, Lee SC, et al. Efficacy and tolerability of pregabalin using a flexible, optimized dose schedule in Korean patients with peripheral neuropathic pain: a 10-week, randomized, double-blind, placebocontrolled, multicenter study. Clin Ther. 2010;32(14):2370-2385.

28. Sabatowski R, Gálvez R, Cherry DA, et al. Pregabalin reduces pain and improves sleep and mood disturbances in patients with post-herpetic neuralgia: results of a randomised, placebo-controlled clinical trial. Pain. 2004;109(1-2):26-35.

29. van Seventer R, Feister HA, Young JP, Stoker M, Versavel M, Rigaudy L. Efficacy and tolerability of twice-daily pregabalin for treating pain and related sleep interference in postherpetic neuralgia: a 13-week, randomized trial. Curr Med Res Opin. 2006;22(2):375-384.

30. Ogawa S, Satoh J, Arakawa A, Yoshiyama T, Suzuki M. Pregabalin treatment for peripheral neuropathic pain: a review of safety data from randomized controlled trials conducted in Japan and in the West. Drug Saf. 2012;35(10):793-806.

31. Vinik A, Emir B, Parsons B, Cheung R. Prediction of pregabalinmediated pain response by severity of sleep disturbance in patients with painful diabetic neuropathy and post-herpetic neuralgia. Pain Med. 2014;15(4):661-670.

32. Parsons B, Li C. The efficacy of pregabalin in patients with moderate and severe pain due to diabetic peripheral neuropathy. Curr Med Res Opin. 2016;32(5):929-937.

33. Ogawa S, Suzuki M, Arakawa A, Yoshiyama T, Suzuki M. [Long-term efficacy and safety of pregabalin in patients with postherpetic neuralgia: results of a 52-week, open-label, flexible-dose study]. Masui. 2010;59(8):961-970. Japanese.

34. Honda M, Murata T, Ebata N, Fujii K, Ogawa S. Treatment patterns of postherpetic neuralgia patients before and after the launch of pregabalin and its effect on medical costs: analysis of Japanese claims data provided by Japan Medical Data Center. J Dermatol. 2017;44(7):767-773.
Journal of Pain Research

\section{Publish your work in this journal}

The Journal of Pain Research is an international, peer reviewed, open access, online journal that welcomes laboratory and clinical findings in the fields of pain research and the prevention and management of pain. Original research, reviews, symposium reports, hypothesis formation and commentaries are all considered for publication.

\section{Dovepress}

The manuscript management system is completely online and includes a very quick and fair peer-review system, which is all easy to use. Visit http://www.dovepress.com/testimonials.php to read real quotes from published authors. 